\title{
Speicher für die Energiewende
}

W. Gawlik

Online publiziert am 30. November 2013

(C) Springer Verlag Wien 2013

Elektrische Energie lässt sich nur sehr schwer speichern. Die Speicherfähigkeit elektrischer Netze selber ist dabei praktisch vernachlässigbar. Zwar sind die von hohen Betriebsströmen durchflossenen Induktivitäten und auf hohe Betriebsspannungen aufgeladenen Kapazitäten im Drehstromsystem mit einem gewissen Energieinhalt verbunden, doch wird dieser während der 20 ms langen Netzperiode bei $50 \mathrm{~Hz}$ Wechselspannung jeweils zweimal mit dem System ausgetauscht. Die mit diesem Vorgang verbundene Ladeleistung ist bei den angestrebten und im Systembetrieb auch erreichten Leistungsfaktoren nahe 1 dann jedenfalls kleiner als die übertragene Wirkleistung. Die im elektrischen Netz gespeicherte Energie würde die Netzlast also gerade für einige wenige Millisekunden decken.

Die in den rotierenden Massen elektrischer Maschinen wie $z$. B. der Generatoren in Kraftwerken gespeicherte Energie, die dafür sorgt, dass sich Ungleichgewichte in Erzeugung und Verbrauch elektrischer Energie in nicht zu steilen Frequenzgradienten und auf sie folgenden Systemzusammenbrüchen äußern, führt zu Systemanlaufzeiten im Bereich 5 bis 10 Sekunden. Speicher zur zeitlichen Entkopplung von in der Regel volatiler erneuerbarer Erzeugung und Verbrauch müssen aber in der Lage sein, wesentlich längere Zeiträume zu überbrücken. Während z. B. mit Pumpspeicherkraftwerken oder durch Einsatz von Demand Side Management eine tageszeitliche Angleichung von Erzeugung und Verbrauch möglich ist, benötigt die Energiewende insbesondere saisonale Langzeitspeicher einer Kapazität, wie sie heute bei weitem nicht zur Verfügung stehen.

Neben den Speicherkraftwerken im alpinen Raum nutzten und nutzen unsere Energiesysteme hauptsächlich die in Form von che- mischer Energie in fossilen Brennstoffen gespeicherte Energie, die je nach Bedarf in thermischen Kraftwerken in elektrische Energie gewandelt wird. Die Energiewende als Abkehr von fossilen Energieträgern erfordert die Substitution durch andere Langzeitspeicher, die zu Zeiten eines Überangebots regenerativer Erzeugungskapazität beladen werden und zu Zeiten, in denen sie nicht verfügbar sind, ihre Energie wieder abgeben können.

Das Potential für Speicher- und Pumpspeicherkraftwerke reicht nicht aus, um diese Kapazitäten sicherzustellen. Für die Energiewende sind deswegen andere Speichertechnologien wie zum Beispiel Power to Gas (P2G) notwendig. Beim P2G-Prozess wird elektrische Energie in Form von Wasserstoff oder synthetischem Methan gespeichert und kann dann über Brennstoffzellen oder in konventionellen Gasturbinen rückverstromt werden. Das Gasnetz verfügt mit den vorhandenen Porenspeichern in Österreich über die Speicherkapazität, um saisonale Schwankungen der volatilen erneuerbaren Energiewandlung auszugleichen. Zur optimalen Integration des P2G-Prozesses ist eine Energieträger-übergreifende Betrachtung des gesamtem Energiesystems und der hybriden Netze (Strom-/ Gasund Fernwärme-Netz) im Universal Grid notwendig.

Dabei stehen saisonale Langzeitspeicher und Kurzzeitspeicher wie Pumpspeicherkraftwerke nicht in Konkurrenz zueinander. Vielmehr führt ein höherer Anteil reversibler Langzeitspeicher im System zu einer höheren Auslastung der Kurzzeitspeicher.
Kurzfassung eines Vortrags bei der 51. Fachtagung der Österreichischen Gesellschaft für Energietechnik im OVE, die am 10. und 11. Oktober 2013 in Graz stattfand.

Gawlik, Wolfgang, Institut für Energiesysteme und Elektrische Antriebe, Technische Universität Wien, Gußhausstraße 25/E370-1, 1040 Wien, Österreich (E-Mail: wolfgang.gawlik@tuwien.ac.at) 